\title{
Die Deutsche Frage und der Brexit. Eine außenpolitische Exemplifizierung, warum Deutschland die Auslösung von Artikel 50 nicht erwägt
}

\author{
Simon Odrowski
}

Online publiziert: 15. Juni 2020

(C) Der/die Autor(en) 2020

Zusammenfassung Während der Brexit nicht zuletzt mit der Wiederherstellung britischer Unabhängigkeit in der Außenpolitik begründet wurde, befürwortet Deutschland die tiefere Integration Europas in diesem sensiblen Bereich nationaler Souveränität. Das Beispiel des EAD zeigt, wie dasselbe Motiv - die Wahrung des nationalen Interesses - diese miteinander kontrastierenden europapolitischen Ansätze erklärt. Außenpolitisch veranschaulicht der EAD damit das generelle Verhältnis zwischen dem europäischen Einigungsprozess und der Deutschen Frage - und wieso Deutschland dem britischen Beispiel eines EU-Austritts nicht folgt.

Schlüsselwörter Deutsche Frage · Deutsche Außenpolitik · Brexit · EAD · Europäische Außenpolitik

\section{The German Question and Brexit. A Foreign Policy Exemplification of Why Germany Does Not Consider Triggering Article 50}

\begin{abstract}
While restoring British independence in foreign policy was one of the main arguments for Brexit, Germany is committed to Europe's deeper integration in this sensitive area of national sovereignty. The EEAS is an example of how the same motivation - the safeguarding of the national interest - drives these contrasting European policy approaches. As a foreign policy example, the EEAS thereby illustrates the general relationship between the European integration process and the German Question - and why Germany does not follow Britain's lead in leaving the EU.
\end{abstract}

Der Beitrag gibt ausschließlich die persönliche Meinung des Autors wieder.

S. Odrowski $(\bowtie)$

Think Tank, Deutsches Zentrum für Luft- und Raumfahrt (DLR), Linder Höhe, 51147 Köln,

Deutschland

E-Mail: simon.odrowski@dlr.de 
Keywords German Question · German Foreign Policy · Brexit · EEAS · European Foreign Policy

\section{Hintergrund: Brexit, Dexit und Außenpolitik}

Als die Brit*innen am 23. Juni 2016 für den Austritt ihres Landes aus der Europäischen Union stimmten, entbrannten Diskussionen um einen möglichen Dominoeffekt. Würde der Brexit das europäische Integrationsprojekt weitere Mitgliedstaaten, gar Mitbegründer wie Italien oder die Niederlande, kosten? Würde Frankreich infiziert und nach der Präsidentschaftswahl 2017 einen Frexit fordern? Die Panik proeuropäischer Strömungen und ein neues Selbstvertrauen europaskeptischer Kräfte brachten zwar allerlei Kofferwörter zur Beschreibung potenzieller Austritte von EU-Mitgliedern hervor. Von einem etwaigen Ausscheiden Deutschlands aus der EU war allerdings kaum die Rede; der Dexit war, wenn überhaupt, nur ein Randphänomen.

Zwar warten alle deutschen Parteien mit Reformvorschlägen für die EU auf, doch das Gros der Politik in Deutschland befürwortet die europäische Integration entschieden; EU-kritischen Ansichten und Vertreter*innen wird kaum Aufmerksamkeit zuteil (Wiesner 2018, S. 296-301). Auch in der deutschen Bevölkerung ist die Zustimmung zu der Aussage, das Land sei ohne EU-Mitgliedschaft für die Zukunft besser gerüstet, mit rund 16\% sehr gering (Europäische Kommission 2019, S. 91). Diese integrationsfreundliche Haltung Deutschlands wurde im Europäischen Konvent 2002/2003 in besonderer Weise deutlich, als im Zuge der Erarbeitung des Vertrags über eine Verfassung für Europa erstmals die Aufnahme einer expliziten Austrittsklausel in das Vertragswerk der EU diskutiert wurde. So war es die deutsche Seite, die ein Amendment Form mit dem Ziel der vollständigen ,Streichung von Art. 46 des Verfassungsentwurfs“ (Meyer 2003, S. 1217) einbrachte. Kritisch antizipierte der den Antrag stellende Jürgen Meyer, Delegierter des Deutschen Bundestages im Konvent, damals unter anderem: „Eine ausdrückliche Austrittsregelung birgt [...] die Gefahr der missbräuchlichen Nutzung durch Europagegner in den Mitgliedstaaten“ (Meyer 2003, S. 1217).

Den jüngsten Verweisen europaskeptischer Kräfte auf Artikel 50 des Vertrags über die Europäische Union - als Nachfolger jenes Artikel 46 - ist das Motiv der Wiederherstellung nationaler Souveränität gemein. So wurde Großbritannien von Europakritiker*innen als leuchtendes Beispiel gefeiert, wie das Joch der EU abgeworfen und die Handlungsfähigkeit des eigenen Landes wiederhergestellt werden könne. Die Deklaration des 23. Juni als ,,independence day“ Großbritanniens durch den ehemaligen Vorsitzenden der UK Independence Party Nigel Farage (zit. n. BBC 2016) spitzt die Argumentation der Brexiteers in einem Begriff zu. Nicht zuletzt galt diese Forderung nach Unabhängigkeit einer außenpolitischen Souveränität: Kontrolle sollte in bereits vergemeinschafteten Bereichen des auswärtigen Handelns, etwa der Handelspolitik, zurückgewonnen und in noch nicht integrierten Feldern erhalten werden. Tatsächlich wurde gerade an seiner Ablehnung der außenpolitischen Integration des Kontinents Großbritanniens Rolle als awkward partner in Europa deutlich (Müller-Brandeck-Bocquet 2019, S. 19). 
Mit diesem speziellen Verhältnis zum europäischen Integrationsprojekt und der nunmehr kompletten Rückabwicklung seiner EU-Mitgliedschaft mag Großbritannien ein besonderer Fall sein. Dass sich beides nicht zuletzt aus einer Ablehnung von Integration im auswärtigen Handeln speist, ist jedoch nicht ganz überraschend, gilt Außenpolitik doch als ,last bastion“ der Nationalstaaten im europäischen Einigungsprozess (Wallace 2005, S. 455). Deutschland allerdings forderte im Verfassungskonvent nicht nur die Streichung einer expliziten Austrittsklausel, sondern setzte sich vielmehr auch ausdrücklich für eine Integration im Bereich der Diplomatie ein. Als wichtigstes nicht militärisches Mittel der Außenpolitik, ein Privileg von Staaten (Wouters und Duquet 2012, S. 33) und ,one of the ways in which states manifest their sovereignty" (Adler-Nissen 2013, S. 186) ist diese ein besonders sensibler Bereich jener letzten Bastion der Nationalstaaten.

So präsentierte im November 2002 der deutsche Diplomat Gunter Pleuger in der Arbeitsgruppe VII External Action des Konvents verschiedene Vorschläge für eine institutionelle Konsolidierung der außenpolitisch-diplomatischen Strukturen der Union (Pleuger 2002). Der deutsche Außenminister Joschka Fischer und sein französischer Amtskollege Dominique de Villepin brachten kurz darauf einen Europäischen Diplomatischen Dienst und einen Europäischen Außenminister ins Spiel (Fischer und de Villepin 2003, S. 4). Diese fanden als Europäischer Auswärtiger Dienst (EAD) und Außenminister der Union Eingang in den Entwurf des Vertrags über eine Verfassung für Europa (2004) und wurden nach dessen Scheitern in den Vertrag von Lissabon übernommen. Der Außenminister der Union wurde dabei in Hoher Vertreter der Europäischen Union für Außen- und Sicherheitspolitik umbenannt (EUV 2016) und entstand durch Zusammenführung der Ämter des ähnlich benannten Hohen Vertreters für die Gemeinsame Außen- und Sicherheitspolitik sowie des Kommissars für Außenbeziehungen. Der EAD wurde mit Beschluss des Rates der Europäischen Union (2010) durch Überführung der einschlägigen Einheiten aus der Kommission sowie aus dem Generalsekretariat des Rates geschaffen und mit Personal aus den diplomatischen Diensten der Mitgliedstaaten verstärkt. Damit entsprach die Art und Weise der institutionellen Konsolidierung europäischer Außenpolitik dem von Deutschland ursprünglich favorisierten ,complete merger of the two offices, their substructures and their areas of responsibility“ (Pleuger 2002, S. 2, eigene Hervorhebung). Neben der Zentrale in Brüssel gehören zum EAD die heute über 140 Delegationen der EU in Drittstaaten - darunter die neueste in London (EAD 2020).

Während Großbritannien also nach Unabhängigkeit strebt, nicht zuletzt in der letzten Bastion Außenpolitik, ist Deutschland mit seiner vehementen Unterstützung des EAD (Adebahr 2015, S. 107; Möller und Rappold 2012, S. 7) Verfechter integrierter Strukturen im sensiblen Bereich der Diplomatie. Der deutschen wie der britischen Europapolitik liegt dabei jedoch, wie im Folgenden auf der Basis von Experteninterviews dargelegt, dieselbe Motivation zugrunde: die Wahrung des nationalen Interesses. Dies zeigen einerseits ein Einblick in die tieferliegenden deutschen Motivationen, einen gemeinsamen diplomatischen Dienst für Europa vorzuschlagen und dessen Gründung zu unterstützen sowie andererseits die Betrachtung des deutschen Ansatzes gegenüber dem EAD seit dessen Einrichtung 2010. Auf diese Weise wird am außenpolitischen Beispiel des EAD nachvollzogen, dass und wieso der 
Brexit und die gleichzeitig ausbleibende Diskussion um einen Dexit kein Widerspruch sind: Geschichte und Geopolitik lassen die räumlich vom Rest Europas abgetrennten Brit*innen die europäische Einigung als Einschränkung ihrer Souveränität und Interessen erfahren. Die Menschen in der Mitte des Kontinents hingegen werden - in ihrem eigenen Interesse - durch das Integrationsprojekt zwar als Deutsche eingehegt, zugleich aber als Europäer*innen mobilisiert. Kern dieser Argumentation ist die historisch-geopolitische Deutsche Frage.

\section{Die europäische Antwort auf die Deutsche Frage}

Als erster diplomatischer Dienst, der nicht von einem Staat geschaffen wurde (Petrov et al. 2012, S. 1), ist der EAD eine Institution sui generis: Der Hybrid ist weder supranational noch intergouvernemental; personell, institutionell wie inhaltlich steht er zwischen den Stühlen von Rat, Kommission und den Mitgliedstaaten. Insofern die EU eine Entität eigener Art ist, scheint es wenig verwunderlich, dass er sich vom Aufbau nationaler Außenministerien signifikant unterscheidet. Besonders im Lichte der skizzierten nationalstaatlichen Bedeutung von Außenpolitik ist vielmehr überraschend, dass der EAD überhaupt existiert - und vom größten und wirtschaftlich stärksten EU-Mitglied vorgeschlagen wurde und mit Nachdruck befürwortet wird.

Tatsächlich wurde Deutschland in den vergangenen zwanzig Jahren immer wieder ein proaktiveres und ausdrücklich selbstbewussteres Argumentieren, teilweise auch Agieren, in der nationalen Außenpolitik attestiert. Dabei besteht jedoch die Kontinuität der multilateralen und europäischen Ausrichtung des auswärtigen Handelns Deutschlands fort (Bulmer und Paterson 2010; Hellmann 2002; Hellmann et al. 2014, S. 103-113). Deutschland, so die gemeinsame Forderung des ehemaligen Außenministers Frank-Walter Steinmeier (2014) und des damaligen Bundespräsidenten Joachim Gauck (2014, S. 118) bei der Münchner Sicherheitskonferenz 2014, solle sich zwar „früher, entschiedener und substanzieller“ einbringen, dies allerdings explizit im multilateralen Rahmen. Wenn das Land als „hegemon“ in Europa charakterisiert wird, erfolgt dies relativierend durch Attribute wie ,embedded“ (Crawford 2007) oder ,reluctant“ (Bulmer und Paterson 2013).

Die zentrale Motivation für diese bewusste europäische Einbettung deutschen Gestaltungswillens wird an deutschem Handeln in Osteuropa besonders deutlich, zuletzt als Deutschland in den Bemühungen um eine Deeskalation der UkraineKrise 2014 auf europäischer Seite die Führungsrolle übernahm (Hellmann 2016, S. 11). Steinmeier sei zum Höhepunkt der Krise von einem osteuropäischen Kollegen als „Herr Ribbentrop“ begrüßt worden, so Rolf Mützenich (Interview 4), Kollege Steinmeiers in der Sozialdemokratischen Partei Deutschlands (SPD). Grund sei, dass ,weiterhin in diesen historischen Kategorien gedacht wird: Die Deutschen vereinbaren etwas mit den Russen, geben Land her und teilen sich sozusagen einen bestimmten europäischen Raum auf“ (Interview 4). Das Beispiel zeigt damit in besonderer Weise, dass deutsche Außenpolitik, gerade wenn sie sich in proaktivem und explizit selbstbewusstem Handeln äußert, eine entscheidende multilaterale Dimension braucht. Wenn Deutschland nachhaltig, wirkungsvoll und fundiert gestalten will, muss es die Interessen von Nicht-EU-Ländern, vor allem aber die seiner EU-Part- 
ner einbeziehen. Während die Verhandlungen zur Deeskalation der Ukraine-Krise selbst im sogenannten Normandie-Format von Deutschland, Frankreich, der Ukraine und Russland geführt wurden, hat Deutschland unter anderem über den EAD versucht, ebenso einen Ausgleich der Interessen der EU-Mitgliedstaaten untereinander zu erreichen (Interview 24).

In der auf deutscher Seite stark verinnerlichten Logik sind eine internalisierte Überzeugung vom europäischen Weg in der Außenpolitik und eine Verfolgung nationaler außenpolitischer Interessen daher kein Widerspruch. Dem europäischen Ansatz Deutschlands liegt damit ein rationalistischer Kern zugrunde. Diesen formuliert ein nicht deutscher EU-Beamter zugespitzt: Gerade die Nachbarschaft werde von Deutschland als Priorität für den EAD gesehen ,as it gives cover to a German Alleingang [sic]“ (Interview 19). Tatsächlich wird Deutschland ein großes Interesse an einem Engagement des EAD gerade in Osteuropa bzw. bezüglich der östlich der EU liegenden Partnerländer der Europäischen Nachbarschaftspolitik, der Östlichen Partnerschaft, attestiert (Interviews 8, 16, 18, 19, 20).

Tieferliegende Gründe der vom Beispiel Osteuropas implizierten spezifischen Rahmenbedingungen deutscher Außenpolitik werden bei einem Vergleich mit dem britischen Ansatz auswärtigen Handelns deutlich. Die Brit*innen würden, wie Joschka Fischer ausführt, außenpolitisch noch viel ungebrochener von der nationalen Interessenebene diskutieren. Den Grund für diesen vom deutschen abweichenden, britischen Diskurs bringt er treffend auf den Punkt: Deutschland habe eine andere geopolitische Lage im Zentrum Europas, eine andere Geschichte und - bedingt durch diese Geschichte - auch eine andere Mentalität (Interview 1).

Während Großbritannien eine Insel mit Zugang zum offenen Meer ist, liegt Deutschland im Herzen Europas, umgeben von heute neun Nachbarn. Diese Mittellage in Europa wirft historisch seit jeher die Deutsche Frage auf: Ist Deutschland - vor allem durch territoriale Fragmentierung - zu schwach, ist es in seiner Handlungsfähigkeit eingeschränkt. Ist Deutschland - insbesondere durch territoriale Einheit - zu stark, stellt es eine Gefahr für seine Nachbarn dar. Beide Mechanismen destabilisieren letztlich Europa wie Deutschland selbst. Wie kann deutsche Handlungsfähigkeit also hergestellt werden, ohne dass Deutschland seine Nachbarn bedroht oder diese sich bedroht fühlen? (Kundnani 2016; Simms 2014)

Die Nachkriegszeit hatte zum ersten Mal eine Antwort auf diese Deutsche Frage in Aussicht gestellt: die politische Integration des europäischen Kontinents. Dass diese die deutsche Geschichte tatsächlich zu einer Erfolgsstory macht, hat die Wiedervereinigung Deutschlands rund zehn Jahre vor dem Europäischen Konvent erneut und in ausdrücklicher Weise gezeigt. Denn die deutsche Einheit wurde für die Nachbarn des Landes durch eine tiefere Integration Europas - und damit Deutschlands in Europa - akzeptabel (vgl. Müller-Brandeck-Bocquet und Rüger 2015, S. 57-60). Die so durch den europäischen Rahmen erst ermöglichte Wiedervereinigung stellte die volle, insbesondere außenpolitische Souveränität Deutschlands wieder her. Diese „Sternstunde“ Deutschlands, so ein Berater der deutschen Delegation im Europäischen Konvent, war zentral für eine allgemeine Unterstützung weiterer Integrationsschritte (Interview 21). Den europäischen Integrationsprozess als Erfolgsmodell Deutschlands bestätigend, schuf die Wiedervereinigung auf deutscher Seite auch die Bereitschaft zur außenpolitischen Integration Europas (Interview 24). Mit sei- 
ner Befürwortung eines gemeinsamen diplomatischen Dienstes wollte dieses jüngst vereinte Deutschland schließlich beweisen, dass es weiterhin abzugeben bereit war (Interview 12).

Das Beispiel der Außenpolitik illustriert so, wie die europäische Integration eine umsichtige Antwort auf die eine Seite der Deutschen Frage - die Phasen territorialer Fragmentierung und eingeschränkter Handlungsfähigkeit - gibt. Deutschland hat gelernt, dass Einheit und vollständige Souveränität des Landes nachhaltig gerade durch Abgabe von Unabhängigkeit möglich werden: Einbettung schafft Handlungsfähigkeit (Haftendorn 2001, S. 436). Mit dieser deutschen Lernerfahrung zu Souveränität kontrastiert jene Großbritanniens, das seit Jahrhunderten nur nationale Einheit sowie außenpolitische Souveränität kennt und über einen langen Zeitraum gar globale Expansion erlebte. Ruprecht Polenz, zur Zeit der Gründung des EAD 2010 amtierender Vorsitzender des Auswärtigen Ausschusses des Bundestages, bewertet aus der Sicht eines deutschen Politikers die - somit ebenfalls historisch bedingte - Auffassung Großbritanniens von Souveränität und unterstreicht so die Gegensätzlichkeit der beiden Verständnisse:

Die Briten haben das Denken aus der nationalstaatlichen Box heraus bisher nie richtig gelernt; das ist ja auch ein Grund, warum sie sich jetzt von der EU verabschieden. Sie denken nach wie vor in vergangenen Kategorien des 19. Jahrhunderts von Souveränität und Nationalstaatlichkeit - aber das ist nicht das Denken, wie wir in Europa weiterkommen (Interview 2).

Doch die Antwort der europäischen Integration auf die Deutsche Frage endet nicht mit der Schaffung von Souveränität und potenzieller Handlungsfähigkeit für Deutschland. Als Antwort auf die andere Seite der Deutschen Frage - die Phasen territorialer Einheit und Stärke - werden deutsche Souveränität und Handlungsfähigkeit durch die europäische Ebene in eingebetteter Form auch mobilisiert. Davon zeugt der auswärtige Dienst der EU unmittelbar, da er Deutschland ermöglicht, außenpolitisch Verantwortung im multilateralen Rahmen zu übernehmen (Interviews 3, 4, 9, 13, 21). Auch Thomas Matussek, unter Fischer Generaldirektor in der Politischen Abteilung des Auswärtigen Amtes und deutscher Botschafter in London, Neu Delhi und bei den Vereinten Nationen, stimmt zu, dass der EAD und die europäische Außenpolitik insgesamt ein angemessener Weg für Deutschland sind, nach der Wiedervereinigung mehr Verantwortung zu übernehmen (Interview 5).

Dieser Ansatz wurde schon wenige Jahre nach der Wiedervereinigung und kurze Zeit vor dem Europäischen Konvent ersichtlich, als gerade Geschehnisse in Osteuropa, damals in dessen Süden, Deutschland in ein Dilemma brachten: Sollte das nunmehr vereinte Land im Herzen Europas in die Konflikte im auseinanderbrechenden Jugoslawien eingreifen? Würden dadurch nicht wieder alte Vorbehalte und Ängste ausgelöst? Angesichts dieser Fragen verbat die Kohl-Doktrin den Einsatz deutscher Soldaten in allen Gebieten, die von der Wehrmacht besetzt gewesen waren. Andererseits: Konnte man, gerade vor dem Hintergrund des Holocausts, der Gewalt und den massiven Menschenrechtsverletzungen tatenlos zusehen? Musste man nicht gerade wegen der eigenen Vergangenheit tätig werden, so die FischerDoktrin? (Simms 2003, S. 402, 413, 2014, S. 494) 
Die deutsche Antwort war schließlich: Eingreifen ja, aber im multilateralen Rahmen. Vor allem musste deutsches Handeln über kurzfristige militärische Eingriffe - im Rahmen der NATO-Operationen - hinausgehen. Aus Fischers Sicht brauchte es für eine langfristige, nicht militärische politische Lösung die EU als handelndes Subjekt. Ein gemeinsamer diplomatischer Dienst der EU würde, so Fischer die damalige Perspektive erinnernd, den institutionellen Rahmen schaffen, um auf dem Balkan pazifizierend wirken zu können, indem den Ländern der Region ein Weg zu einer Beitrittsperspektive zur EU eröffnet würde (Interview 1). Die europäische Einbettung Deutschlands zur Schaffung von Handlungsfähigkeit sowie die Einbettung jener Handlungsfähigkeit selbst exemplifizieren somit außenpolitisch, wie Europa als Antwort auf die Deutsche Frage dient.

\section{Die Deutsche Frage als Antwort für Europa}

Das europäische Einigungsprojekt und die Deutsche Frage sind darüber hinaus noch auf eine zweite Weise miteinander verbunden. Denn aus deutscher Sicht fungieren umgekehrt auch Erfahrungen mit - beiden Seiten - der Deutschen Frage als Antwort für Europa. Anders formuliert: Nationale Erfahrungen begründen einerseits die deutsche Disposition für die Integration und ihre Fortentwicklung, auch in der Außenpolitik, mit und prägen andererseits deutsche Entwürfe für die konkrete Ausgestaltung der Einigung. Deutschlands Haltung gegenüber dem EAD bietet hierfür zwei anschauliche Beispiele: Zum einen wird ersichtlich, dass ein durch die eigene Historie bedingter föderalistischer Ansatz deutscher Politik auch in der Europapolitik Deutschlands zum Tragen kommt. Zum anderen zeitigt die deutsche Geschichte ein Selbstverständnis Deutschlands als Zivilmacht, die per definitionem prosupranational eingestellt ist und zudem dieses Verständnis auch auf die europäische Ebene übertragen möchte.

Die Logik des föderalistischen Ansatzes der Bundesrepublik erschließt sich, beginnt man bei der Sicht deutscher Politiker und Beamt*innen in Berlin und Brüssel, dass der EAD dazu dient, durch eine gemeinsame Stimme Europas mehr Einfluss in der internationalen Politik zu haben (Interviews 2, 4, 6, 8, 10, 14). Dieser Perspektive liegt die Überzeugung zugrunde, dass das Gewicht jedes europäischen Landes, selbst des größten EU-Mitgliedstaates, auf der Bühne der Weltpolitik für sich genommen relativ klein ist. Diese deutsche Sichtweise wird auch an den zwei bereits skizzierten Ereignissen in Ost- und Südosteuropa deutlich.

So waren die - vom EAD ausgearbeiteten - Sanktionen gegenüber Russland in der Ukraine-Krise aus deutscher Sicht erst durch die gemeinsame europäische Ebene sinnvolles außenpolitisches Mittel (Interview 20). Dieses Beispiel reflektiere die realpolitische deutsche Perspektive, dass eine gemeinsame Außenpolitik viel effektiver als nationale Vorstöße sei (Interview 21). Im Gegensatz zur UkraineKrise hatten die Konflikte im ehemaligen Jugoslawien in den 1990er Jahren noch die fehlende Geschlossenheit der EU offenbart (Interview 12) und gezeigt, dass es ihr an einem geostrategischen, diplomatischen Element mangelte (Interview 21): Als einzelne Länder hatten die europäischen Staaten nicht einmal ausreichend Gewicht für eine Lösung eines Konflikts an der Türschwelle des Kontinents. Eine Chance, 
in der Region Frieden zu schaffen, wäre nur im gemeinschaftlichen europäischen Rahmen gegeben.

Die Idee des damaligen Außenministers Fischer war daher - in Anlehnung an die Politik der Westalliierten gegenüber Deutschland ab 1945 - einen Plan zu haben, der etwas verspricht. In dem Fall war das Versprechen die Teilhabe am gemeinsamen Europa. Aus Fischers Sicht konnte keine Nation auf nationaler Grundlage, sondern nur die EU als Ganze ein solches Versprechen machen. Dafür bedurfte es einer entsprechenden apparativen Ausstattung, das heißt eines gemeinsamen auswärtigen Dienstes als entscheidendes Instrument der gemeinsamen Außen- und Sicherheitspolitik (Interview 1).

Natürlich war die Realität der Balkan-Konflikte für Großbritannien dieselbe wie für Deutschland. Entsprechend sah auch die erste Hohe Vertreterin und Britin Catherine Ashton den Balkan als ,the birthplace of EU foreign policy“ (Ashton 2010): Die Einsicht, dass Europa gemeinsam agieren musste, war eine geteilte. Auch aus britischer Sicht war der EAD daher im Lichte des schwindenden Einflusses der europäischen Nationalstaaten zu sehen (Pilegaard und Kluth 2012). Allein, der britische Ansatz im EAD war deutlich funktionalistisch und fragte nach konkreten capabalities, die das Vereinigte Königreich selbst nicht besitzt, um nationale Interessen zu wahren oder zu verstärken (Interview 24). Für die Brit*innen war der EAD eher ein „Transmissionsriemen“ (Interview 13) bzw. „Vehikel“ (Interview 15) zur Übertragung bestimmter Interessen auf die europäische Ebene (Interview 6). Letztere sollte also nur in jenen konkreten Fällen aktiv werden, in denen es auf nationaler Ebene an Fähigkeiten oder Einfluss mangelt.

Dieser instrumentelle Ansatz steht der generellen Disposition Deutschlands für integrierte außenpolitische Strukturen gegenüber. Während sich Großbritannien mittlerweile sogar gänzlich better off out wähnt, sieht sich Deutschland angesichts der Globalisierung per se als Teil der größeren Entität EU besser aufgehoben (Interviews 21, 23). An der ursprünglichen Diskussion um den EAD wird die föderalistische Orientierung offensichtlich, die dieser deutschen Haltung wesentlich mit zugrunde liegt und so auch den Unterschied zum britischen Ansatz miterklärt: Andere EU-Mitgliedstaaten, die auf europäischer Ebene nicht gleichermaßen wie Deutschland auf föderale Strukturen bedacht seien, seien überrascht gewesen, wie stark das Land gemeinsame außenpolitische Strukturen während des Europäischen Konvents befürwortet habe (Interview 11).

Dieser föderale Ansatz in der Europapolitik ist vor dem Hintergrund des eigenen politisch-institutionellen Aufbaus Deutschlands zu sehen. Während die europäische Integration einen disruptiveren Effekt auf „simple politites“ wie Großbritannien hat (Schmidt 2005), ist Deutschland nicht nur, so die Annahme eines institutional fit (Wiesner 2018, S. 295), besser für die Integration in die EU gerüstet. Tatsächlich ist das eigene politisch-administrative Set-up Deutschlands geradezu prädestiniert für die Befürwortung gemeinsamer Strukturen auf übergeordneter europäischer Ebene. In diesem Zusammenhang ist die Wahrnehmung Cesare Onestinis, seit 1995 für die EU tätig und unter anderem von 2014 bis 2017 stellvertretender Leiter der EUDelegation in Indien, bemerkenswert. Dieser hatte den Eindruck, Deutschlands Vorschlag und starke Unterstützung eines gemeinsamen diplomatischen Dienstes sei ,in line with the German approach to the EU, especially in the Convention timeframe." 
Er führt fort: „The EU was seen as a new kind of layer in the federal approach that Germany has to policy making. And in the German system, foreign relations belong to the upper levels“ (Interview 17). Im Gegensatz zur britischen Selektivität ist aus einer solchen Perspektive das grundsätzliche Betreiben von Außenpolitik auf europäischer Ebene der logische Weg: Eine „Kleinstaaterei“ mache aus deutscher Sicht, so ein Berater der deutschen Delegation im Europäischen Konvent, in der globalisierten Welt des 21. Jahrhunderts keinen Sinn (Interview 21).

Der Dysphemismus Kleinstaaterei deutet dabei wieder auf die eine Seite der Deutschen Frage - die Phasen territorialer Spaltung und eingeschränkten Handlungsfähigkeit - und damit die geschichtlich-geopolitische Bedingtheit dieses föderalistisch geprägten Ansatzes hin. Zuletzt im 19. Jahrhundert war das Land ein Flickenteppich, an den Rändern durch unterschiedliche Territorialansprüche und politische Zugehörigkeiten teilweise ausgefranzt. Während das British Empire gerade durch eine aktive Außenpolitik in Form weltweiter Expansion entstand, wurde das Deutsche Reich und erst damit eine gesamtdeutsche Außenpolitik - durch Zusammenschluss vieler einzelner deutscher Staaten geboren. Zur Schaffung von Handlungsfähigkeit gehört Außenpolitik in der historisch bedingten deutschen Logik daher auf die höchstmögliche Ebene.

Für eine zweite Erfahrung aus der Deutschen Frage, die den deutschen Ansatz in der Europapolitik mitbestimmt, spielen hingegen die Lehren aus den Phasen territorialer Einheit und Stärke - der anderen Seite der Deutschen Frage - eine entscheidende Rolle. Diese Perioden waren regelmäßig von einer militaristischen Ausrichtung deutscher Außenpolitik geprägt. Zuletzt die derart beschaffenen Politiken des Wilhelminischen sowie des Dritten Reichs führten zu katastrophalen Konsequenzen für Europa und Deutschland selbst. Mit dem raschen Aufschwung Westdeutschlands zur drittgrößten Volkswirtschaft der Welt nach dem Zweiten Weltkrieg, aber insbesondere seit der Wiedervereinigung bedurfte es daher im nationalen wie europäischen Interesse einer neuen Definition deutscher Macht.

Nicht nur aus akademischer Sicht wurde Deutschland als Zivilmacht beschrieben (Maull 1990). Auch von deutschen Entscheidungsträger*innen selbst wurde der Begriff zur Charakterisierung dessen, was Deutschland nunmehr ist oder sein sollte, aufgegriffen (Maull 2014, S. 128-130). Die mit dem Konzept der Zivilmacht verbundene Hinwendung zu Kooperation, zu einer Präferenz für nicht militärische Mittel und einem Einsatz von Streitkräften nur als Ultima Ratio sowie schließlich zu einer Bevorzugung supranationaler Strukturen (Maull 1990, S. 92) beschreiben dabei als Lehren aus der ersten Hälfte des 20. Jahrhunderts das Gegenteil eines hegemonialen, militaristischen Deutschlands, das seine Nachbarn bedroht. Die deutsche Unterstützung der Vertiefung der europäischen Außenpolitik um die Jahrtausendwende ist gerade auch im Lichte dieses Selbstverständnisses Deutschlands als Zivilmacht zu sehen (Interview 24). Einerseits erklärt die in der Identität als Zivilmacht angelegte Orientierung auf Multilateralität und Supranationalität die deutsche Disposition für außenpolitische Integration daher maßgeblich mit.

Andererseits veranschaulicht der EAD, dass dieses Zivilmacht-Verständnis aus deutscher Sicht auch auf die EU übertragen werden soll. Denn der EAD wird als entscheidender Schritt hin zu einer Zivilmacht Europa gesehen, insofern er das zweite Element des Konzepts - den möglichst umfassenden Verzicht auf militärische 
Mittel in der Außenpolitik - auf der europäischen Ebene fest verankern soll. Um eine Zivilmacht sein zu können, müssten, so die Forderung von deutscher Seite, die militärischen und die zivilen Strukturen der Krisenprävention und des Konfliktmanagements der EU bei der Schaffung des EAD zusammengeführt werden. So soll insbesondere eine Loslösung der militärischen capabilities vermieden werden. Diese Konzeption betonten bei Gründung des EAD 2010 alle an der schwarz-gelben Regierung beteiligten Koalitionspartner sowie die Oppositionspartei Bündnis 90/Die Grünen (Deutscher Bundestag 2010a, S. 3, 2010b, S. 4749-4750).

Dass die Integration der zivilen und militärischen Strukturen in den EAD zudem auch von der SPD befürwortet wurde, ,weil wir auch auf nationaler Ebene stärkere Verschränkungen in diesem Bereich anstreben“ (Interview 4), zeugt sogar unmittelbar davon, dass nationale Ansätze als Vorbild für die europäische Ebene gesehen werden. Wie Frithjof Schmidt, Mitglied des Deutschen Bundestages seit 2009 und zuvor von 2004 bis 2009 Mitglied des Europäischen Parlaments, am Beispiel der Konfliktprävention verdeutlicht, kontrastiert und konkurriert der deutsche Ansatz dabei durchaus mit Konzeptionen anderer EU-Mitgliedstaaten: „Natürlich ist der EAD eine Möglichkeit, Überlegungen eines Konzepts der Konfliktprävention, das sich von einem französischen oder englischen Verständnis von Außenpolitik als Politik der Machtprojektion sehr unterscheidet, in die europäische Debatte einzubringen“ (Interview 3).

\section{Deutsche Fragen und europäische Antworten}

Einerseits lassen sich somit am Beispiel des deutschen Vorschlags für einen gemeinsamen auswärtigen Dienst sowie dem Diskurs um dessen Aufbau elementare, in der Deutschen Frage fußende Grundmotive Deutschlands für die Unterstützung der europäischen Integration nachvollziehen. Am folgenden Blick auf die Zeit seit der Gründung des EAD 2010 wird andererseits die deutsche Perspektive ersichtlich, dass dieser dabei helfen kann, umsichtig und effektiv Antworten auf spezifische deutsche Fragen zu geben. Damit wird die deutsche Sicht illustriert, wie konkrete außenpolitische Interessen durch eine europäische Dimension besser als rein national und im Einklang mit den Anliegen der Partner Deutschlands vertreten werden können. Zwar sah auch Großbritannien im EAD die Möglichkeit, bestimmte nationale Interessen besser verfolgen zu können. Im Gegensatz zur deutschen Haltung war die britische Sicht auf den EAD jedoch auch maßgeblich von einer Wahrnehmung des Dienstes als potenzielles „Trojan horse“ für eine weitere Integration im Bereich der Außenpolitik geprägt (Fiott 2015, S. 78). Im Falle Deutschlands wie auch Großbritanniens wirken die jeweiligen historischen Erfahrungen damit bis hin zur konkreten tagesaktuellen Politik fort.

Ein besonders anschauliches Beispiel für die deutsche Perspektive ist das von Deutschland begrüßte und gewünschte Engagement des EAD in den Verhandlungen mit Iran um dessen Atomprogramm (Interviews 4, 13, 15). Zunächst hatte Deutschland national die Initiative für Gespräche mit dem Land ergriffen, diese später an die europäische Ebene übergeben und die EU schließlich als führenden Akteur zur Problemlösung priorisiert. Das deutsche Selbstverständnis war das eines Wegbereiters, 
der sich sodann in den Dienst des Europäischen stellt (Interview 23). So wurden die Verhandlungen 2003 noch von den nationalen Außenministern Fischer, de Villepin und deren britischem Amtskollegen Jack Straw initiiert. Später wurde Javier Solana als Hoher Vertreter der EU mit in die Gespräche einbezogen; nicht zuletzt weil sich andere europäische Länder, vor allem Italien und Spanien, aus den Verhandlungen ausgeschlossen fühlten (Interviews 1, 4, 23, 24). $\mathrm{Zu}$ einem diplomatischen Erfolg geführt wurden die Verhandlungen schließlich vor allem mit Hilfe der Hohen Vertreterin Federica Mogherini und dem EAD (Interview 1). Dieser Fall charakterisiert dabei einen benevolent bilateralism: Stößt Deutschland Politik auf der nationalen Ebene an und übergibt dann an die europäische, erfolgt dies in der Annahme, auch national im europäischen Interesse zu handeln (Interview 25).

Diese Übertragung der Federführung von der nationalen an die europäische Ebene wurde durch einen bedeutenden personellen Transfer komplementiert: Als Mitarbeiterin Fischers war die deutsche Diplomatin und heutige EAD-Generalsekretärin Helga Schmid zunächst als nationale Vertreterin in Teheran. Schließlich war sie von 2011 bis 2016 als Stellvertretende Generalsekretärin für Politische Angelegenheiten des EAD und damit als Aide-de-camp der Hohen Vertreterin beim Abschluss des Abkommens dabei. Damit war die Deutsche die einzige Person im westlichen Verbund, die von Anfang bis Ende an den Verhandlungen beteiligt gewesen ist (Interview 1). Das Beispiel der Verhandlungen mit Iran deutet dabei auf zwei Mechanismen hin, wie die europäische Ebene der Bearbeitung außenpolitischer Anliegen Deutschlands dient.

Erstens bietet aus deutscher Sicht ein Handeln auf europäischer Ebene gegenüber einem (rein) nationalen Vorgehen Vorteile, indem es nationale Außenpolitik komplementiert bzw. fortsetzt. Das Beispiel der Verhandlungen mit Iran deutet dabei zwar an, dass dies auch für Großbritannien gelten sollte. Dennoch war die britische Wahrnehmung der Einbindung der europäischen Ebene meist eher, dass auf diese Weise Kompetenzen aus der Hand gegeben werden (Interview 15). Schon 2011 warnte der britische Außenminister William Hague seine Diplomat*innen weltweit vor einem möglichen competence creep des EAD (Fiott 2015, S. 80). Die damit kontrastierende deutsche Haltung, die dem gegenüber stärker auf den Mehrwert des EAD fokussiert ist, wird an zwei weiteren Bereichen, in denen Deutschland an einem Engagement des EAD besonders interessiert ist, deutlich: die Afrikapolitik sowie die Krisenprävention bzw. Gemeinsame Sicherheits- und Verteidigungspolitik (GSVP).

Vor allem wegen der verstärkten Migration nach Europa ist Afrika in den vergangenen Jahren in den Fokus deutscher Außenpolitik gerückt (Oswald 2018; Rinke 2016) und zu einer deutschen Priorität für den EAD geworden (Interviews 6, 8, 14, 15). In diesem Handlungsfeld bietet vor allem die Expertise des EAD und der EU-Partner (im EAD) einen deutlichen Mehrwert für Deutschland. Ein deutscher EAD-Beamter erläutert, dass einer der Vorteile des EAD sei, dass der Dienst auf das Know-how ehemaliger Kolonialmächte bei der Formulierung von Interessen bezüglich bestimmter Länder zurückgreifen könne. Gleichzeitig sei er jedoch unbefangener, insofern die EU als solche nicht vollständig mit jenen historischen Verbindungen zu den meisten ehemals kolonisierten Ländern identifiziert werde. Dies könne auch für Deutschland ein Vorteil sein (Interview 11). Die Migrationspartnerschaften mit 
zahlreichen afrikanischen Staaten konnten beispielsweise nur mit dem Gewicht und der Erfahrung der anderen EU-Mitgliedstaaten, der Kommission sowie des EAD ausgehandelt werden (Interview 10).

Die Bedeutung der europäischen Dimension für deutsche Außenpolitik wird weiterhin an Deutschlands Interesse an einem Engagement des EAD in der - insbesondere zivilen - GSVP (Interviews 9, 10, 20) sowie der zivilen Krisenprävention und Konfliktbewältigung (Interviews 3, 4) ersichtlich. So seien die GSVP-Missionen, etwa die Ausbildungsmissionen in Mali oder am Horn von Afrika, etwas, das man auf allein nationaler Ebene gar nicht umsetzen könne bzw. wolle (Interviews 14, 20). Außerdem profitiere Deutschland durch die europäische Ebene auch von der Expertise anderer Staaten in der Krisenprävention (Interview 14). Vor allem aber dürfte an der Ablehnung rein nationaler Initiativen in diesem Bereich die deutsche Präferenz deutlich werden, einer Verantwortung zur Friedenswahrung im multilateralen Rahmen gerecht werden zu wollen (Interviews 4, 9, 13, 21). Damit tritt die bereits genannte, historisch bedingte und bewusste Selbsteinbettung deutschen Handelns wieder zu Tage. Dem gegenüber ist Großbritannien bei der tatsächlichen Umsetzung im Bereich der GSVP gerade deshalb viel zurückhaltender oder enthält sich dieser gar gänzlich, weil nicht zu viele nationale Kräfte von der EU gebunden werden sollen (Interview 20).

Zweitens zeigt das Beispiel der Verhandlungen mit Iran, dass über die Entsendung diplomatischen Personals der Mitgliedstaaten in den EAD die Möglichkeit besteht, Einfluss auf die gemeinsame Stimme zu nehmen, die Deutschland mit dem Vorschlag des EAD angestrebt hat. Dies wird auch an den zwei EU-BotschafterPosten des deutschen Diplomaten Markus Ederer noch einmal deutlich. Ederer war zunächst von 2011 bis 2014 EU-Botschafter in Peking. Dabei sind die Beziehungen zu China nicht nur eine außenpolitische Priorität Deutschlands, sondern gerade deshalb auch eine deutsche Priorität für den EAD, weil hier der gemeinsame Einfluss größer als jener Deutschlands allein ist (Interview 8, 15). Insofern war Ederers Stelle ein herausragender (Interview 2) bzw. wichtiger Posten (Interview 19), um den sich das Auswärtige Amt aktiv bemüht hat (Interview 10, 23). Ganz im Sinne des Selbstverständnisses als „Impulsgeber“ der Politik der EU gegenüber Russland (Böttger 2016, S. 409) wurde auch die 2017 erfolgte Ernennung Ederers zum EUBotschafter in Moskau als eindeutig und umfassend im deutschen Interesse liegend bewertet (Interviews 6, 9, 10, 22, 24).

Auch jenseits der beiden Posten Ederers ist Deutschland daran interessiert (Interviews 6, 13) und damit erfolgreich (Interviews 7, 9, 10, 22, 25), Schlüsselpositionen im EAD zu besetzen. Auch wenn ebenso Großbritannien an wichtigen EAD-Posten für seine Diplomat*innen interessiert war, gab es im Foreign and Commonwealth Office ein starkes Bewusstsein dafür, dass gerade hochqualifizierte Beamt*innen zu wertvoll sind, um sie dem EAD zu überlassen (Fiott 2015, S. 86). Deutsche Diplomat*innen auf zentralen EAD-Posten sind dabei weniger ein Mittel für das blanke Durchdrücken deutscher Positionen. Vielmehr dient die Überlassung deutschen Personals der von Deutschland im eigenen Interesse befürworteten Europäisierung der Politik. So ist die Haltung bei der Entsendung von Personal des Auswärtigen Amtes ,an example of real-life crossloading“: Sie ermöglicht das Einbringen deutscher 
Perspektiven in die europäische Außenpolitik und trägt zugleich zur Europäisierung von policies bei (Adebahr 2015, S. 116).

Das Anliegen einer Besetzung von Schlüsselposten wird vom Ziel einer angemessenen quantitativen presence allemand im EAD komplementiert (Interviews 6, $7,8,9,13)$. Die entsprechende personalwirtschaftliche Aufstellung des Auswärtigen Dienstes Deutschlands als zentrale Voraussetzung dafür, dass Deutschland überhaupt ausreichend Personal für den EAD stellen kann, wird dabei entsprechend häufig problematisiert (Interviews 2, 6, 7, 13, 23). Doch auch die Logik der zahlenmäBig adäquaten Repräsentanz im EAD wird der Europäisierung deutschen Handelns und Denkens unterworfen: Eine mit dem Anteil Deutschlands an der EU-Bevölkerung vollständig korrespondierende Personalpräsenz von etwa $16 \%$ würde vor allem kleinere Länder benachteiligen (Interviews 2, 10, 13). Auch insgesamt würde ein zu starkes Drängen bei der Frage der quantitativen Vertretung deutschen Personals von seinen EU-Partnern wohl nicht positiv aufgenommen (Interviews 23, 24). Diese deutsche Zurückhaltung wird insbesondere bei einem Vergleich mit Großbritannien deutlich: Vor Beginn des durch den Brexit bedingten Abbaus seines Personals stellte Großbritannien $60 \%$ mehr nationale Diplomat*innen im EAD als das bevölkerungsmäßig deutlich größere Deutschland (EEAS 2017, S. 50). Deutsche Personalpolitik im EAD zielt daher insgesamt zwar darauf ab, deutsche außenpolitische Prioritäten und deutsche Prioritäten für den EAD zu stützen, dabei aber gleichzeitig die Interessen der EU-Partner - im eigenen Interesse - explizit und aktiv zu respektieren.

\section{Resümee: Artikel 50 und die Deutsche Frage}

Keine Handlung ist ohne Alternative - auch Deutschland könnte Artikel 50 auslösen und dem europäischen Integrationsprojekt Lebewohl sagen. Der deutsche Vorschlag und die deutsche Unterstützung gemeinsamer diplomatischer Strukturen aber exemplifiziert außenpolitisch, weshalb Deutschland dem britischen Beispiel nicht folgt. Denn ein Dexit wäre nicht nur ein Abschied von der EU, sondern auch ein Abgesang auf die Erfolgsgeschichte Deutschlands in Europa. Der vier Jahre lang drohende und schließlich gerade vollzogene Brexit scheint nicht nur die größte Geschlossenheit zwischen den (übrigen) EU-Staaten der letzten knapp zwei Jahrzehnte hergestellt zu haben. Der Austritt Großbritanniens und die turbulenten Entwicklungen dort haben offenbar vielmehr, nicht zuletzt in Deutschland, die Überzeugung gestärkt, dass ein Ausscheiden aus dieser Union nicht im nationalen Interesse sein kann.

Nicht, dass man sich in Deutschland über den EU-Austritt Großbritanniens freuen würde - er wird bedauert. Doch der deutschen Logik konsequent folgend, wird nunmehr sogar versucht, im Brexit eine Chance zu sehen, die Außenpolitik der EU kohärenter werden zu lassen und ihre Integration voranzutreiben. Denn der Weggang des awkward partner könnte Integrationsbarrieren verringern und Potenziale eröffnen. Beginnend mit einem gemeinsamen Schreiben des deutschen Außenministers Steinmeier und seines französischen Kollegen Jean-Marc Ayrault 2016, nur vier Tage nach dem Brexit-Referendum, hat Deutschland zusammen mit Frankreich bereits zahlreiche Vorschläge zur Verbesserung der Gemeinsamen Außen- und Sicherheitspolitik sowie der GSVP vorgebracht (Müller-Brandeck-Bocquet 2019, S. 20-22). 
Außenpolitik, insbesondere Diplomatie, ist Kernbereich nationaler Souveränität. Die konsequente und fortgesetzte Bereitschaft, in diesem Bereich Kompetenzen und Fähigkeiten abzugeben, selbst in Zeiten nationaler Rückbesinnungen überall in Europa, exemplifiziert daher in besonderer Weise die deutsche Internalisierung des europäischen Weges. Indem der europäische Integrationsprozess eine Antwort auf die Deutsche Frage bietet, indem umgekehrt Erfahrungen mit der Deutschen Frage zur europäischen Integration prädestinieren sowie deutsche Vorstellungen von Europa mit formen und indem schließlich die Wahrung konkreter deutscher Interessen im europäischen Konzert gesucht wird, wird die realpolitische, rationalistische Komponente dieser Lernerfahrung ersichtlich.

Dieses geopolitisch-historisch geprägte, rationalistische Element der europäisierten Außenpolitik Deutschlands löst die konzeptionelle und empirische Spannung zwischen dem normativ von Europa überzeugten Musterknaben der Integration und einem offenkundig auch interessengeleiteten Deutschland. Anders formuliert: Die deutsche Ablehnung eines Austritts aus der EU speist sich gerade deshalb aus einer tiefen Überzeugung vom europäischen Weg, weil dieser maßgeblich im deutschen Interesse liegt und damit sein Beschreiten rationalistisch ist. Denn die europäische Integration hegt die Menschen im Herzen des Kontinents als Deutsche ein und mobilisiert sie zugleich als Europäer*innen - in ihrem eigenen nationalen Interesse.

\section{Interviews}

Interview 1: Joschka Fischer, Cambridge (UK), 23. Mai 2017

Interview 2: Ruprecht Polenz, Telefon, 16. Mai 2017

Interview 3: Frithjof Schmidt, Berlin, 01. Juni 2017

Interview 4: Rolf Mützenich, Berlin, 30. Mai 2017

Interview 5: Thomas Matussek, Berlin, 29. Mai 2017

Interview 6: Mitarbeiter des Auswärtigen Amtes, Berlin, 31. Mai 2017

Interview 7: Mitarbeiter des Auswärtigen Amtes, Berlin, 31. Mai 2017

Interview 8: Mitarbeiter des Auswärtigen Amtes, Berlin, 31. Mai 2017

Interview 9: Mitarbeiter des Auswärtigen Amtes, Berlin, 02. Juni 2017

Interview 10: Mitarbeiter des Auswärtigen Amtes, Telefon, 19. Juni 2017

Interview 11: deutscher EAD-Mitarbeiter, Brüssel, 06. Juni 2017

Interview 12: deutscher EAD-Mitarbeiter, Brüssel, 06. Juni 2017

Interview 13: deutscher EAD-Mitarbeiter, Brüssel 08. Juni 2017

Interview 14: deutscher EAD-Mitarbeiter, Telefon, 19. Juni 2017

Interview 15: deutscher EAD-Mitarbeiter, Telefon, 23. Juni 2017

Interview 16: Eamon Gilmore, Cambridge (UK), 17. Mai 2017

Interview 17: Cesare Onestini, Telefon, 25. Mai 2017

Interview 18: nicht deutscher EAD-Mitarbeiter, Telefon, 19. Juni 2017

Interview 19: ehemaliger nicht deutscher EU-Beamter, E-Mail, 27-29. Mai 2017

Interview 20: Mitarbeiter des Bundesministeriums der Verteidigung, Berlin, 30. Mai 2017

Interview 21: Berater der deutschen Delegation im Europäischen Konvent, persönliches Gespräch 
Interview 22: Nicolai von Ondarza, Berlin, 01. Juni 2017

Interview 23: Ronja Kempin, Berlin, 29. Mai 2017

Interview 24: Niklas Helwig, Telefon, 16. Mai 2017

Interview 25: Cornelius Adebahr, Telefon, 04. Juli 2017

Danksagung Dieses Essay basiert in wesentlichen Teilen auf Forschung, die ohne die Unterstützung weiterer Personen nicht möglich gewesen wäre. Ein besonderer Dank geht daher vor allem an die Interviewpartner*innen. Außerdem bedanke ich mich ausdrücklich bei Prof. Brendan Simms, Prof. Thomas Jäger, Prof. Christopher Clark, Prof. Christopher Hill, Dr. Nick Wright, Dr. Julie Smith, Dr. Geoffrey Edwards, Dr. Karolina Pomorska, Dr. Tobias Etzold, Friderike Uphoff und Stefan Odrowski für unterschiedliche Formen der Unterstützung des Forschungsprozesses.

Funding Open Access funding provided by Projekt DEAL.

Open Access Dieser Artikel wird unter der Creative Commons Namensnennung 4.0 International Lizenz veröffentlicht, welche die Nutzung, Vervielfältigung, Bearbeitung, Verbreitung und Wiedergabe in jeglichem Medium und Format erlaubt, sofern Sie den/die ursprünglichen Autor(en) und die Quelle ordnungsgemäß nennen, einen Link zur Creative Commons Lizenz beifügen und angeben, ob Änderungen vorgenommen wurden.

Die in diesem Artikel enthaltenen Bilder und sonstiges Drittmaterial unterliegen ebenfalls der genannten Creative Commons Lizenz, sofern sich aus der Abbildungslegende nichts anderes ergibt. Sofern das betreffende Material nicht unter der genannten Creative Commons Lizenz steht und die betreffende Handlung nicht nach gesetzlichen Vorschriften erlaubt ist, ist für die oben aufgeführten Weiterverwendungen des Materials die Einwilligung des jeweiligen Rechteinhabers einzuholen.

Weitere Details zur Lizenz entnehmen Sie bitte der Lizenzinformation auf http://creativecommons.org/ licenses/by/4.0/deed.de.

\section{Literatur}

Adebahr, C. (2015). Germany: The instinctive integrationist occasionally going intergovernmental. In R. Balfour, C. Carta, \& K. Raik (Hrsg.), The European External Action Service and national foreign ministries. Convergence or divergence? (S. 107-120). Farnham: Ashgate.

Adler-Nissen, R. (2013). Bourdieu in international relations: Rethinking key concepts in IR (New International Relations). Abingdon: Routledge.

Ashton, C. (2010, 10. März). Joint debate on foreign and security policy. Speech/10/82. European Commission. http://europa.eu/rapid/press-release_SPEECH-10-82_en.pdf. Zugegriffen: 01. Dez. 2019.

BBC - British Broadcasting Corporation. (2016, 24. Juni). EU referendum: Farage declares 'independence day'. https://www.bbc.com/news/av/uk-politics-eu-referendum-36613295/eu-referendumfarage-declares-independence-day. Zugegriffen: 01. Dez. 2019.

Böttger, K. (2016). Deutschland, die Östliche Partnerschaft und Russland. In K. Böttger, \& M. Jopp (Hrsg.), Handbuch zur deutschen Europapolitik (S. 407-420). Baden-Baden: Nomos.

Bulmer, S., \& Paterson, W.E. (2010). Germany and the European Union: From 'tamed power' to normalized power? International Affairs, 86(5), 1051-1073.

Bulmer, S., \& Paterson, W.E. (2013). Germany as the EU's reluctant hegemon? Of economic strength and political constraints. Journal of European Public Policy, 20(10), 1387-1405.

Crawford, B. (2007). Power and German foreign policy. Embedded hegemony in Europe (New Perspectives in German Studies). Basingstoke, New York: Palgrave Macmillan.

Deutscher Bundestag. (2010a, 09. Juni). Antrag der Fraktionen der CDU/CSU und FDP. Einen effizienten und schlagkräftigen Europäischen Auswärtigen Dienst schaffen. Drucksache 17/1981. http://dip21. bundestag.de/dip21/btd/17/019/1701981.pdf. Zugegriffen: 13. Jan. 2020.

Deutscher Bundestag. (2010b, 10. Juni). Stenografischer Bericht. 46. Sitzung. Plenarprotokoll 17/46. http:// dipbt.bundestag.de/doc/btp/17/17046.pdf. Zugegriffen: 13. Jan. 2020. 
EAD - Europäischer Auswärtiger Dienst. (2020, 01. Feb.). Vereinigtes Königreich: Erklärung des Hohen Vertreters zur Eröffnung der EU-Delegation in London. https://eeas.europa.eu/headquarters/ headquarters-homepage/73790/node/73790_de. Zugegriffen: 03. Feb. 2020.

EEAS - European External Action Service. (2017). EEAS human resources. Annual report 2016. https://eeas.europa.eu/sites/eeas/files/eeas_human_resources_reports_2016.pdf. Zugegriffen: 16. März 2020.

Europäische Kommission. (2019). Standard-Eurobarometer 91. Bericht. Die öffentliche Meinung in der EU. https://ec.europa.eu/commfrontoffice/publicopinion/index.cfm/ResultDoc/download/Document Ky/88544. Zugegriffen: 01. Dez. 2019.

EUV - Vertrag über die Europäische Union, Art. 18, 27 (2016).

Fiott, D. (2015). 'Our man in Brussels'. The UK and the EEAS: Ambivalence and influence. In R. Balfour, C. Carta, \& K. Raik (Hrsg.), The European External Action Service and national foreign ministries. Convergence or divergence? (S. 75-88). Farnham, Burlington: Ashgate.

Fischer, J., \& de Villepin, D. (2003, 15. Jan.). Deutsch-französischer Beitrag zum Europäischen Konvent über die institutionelle Architektur der Union (Berlin und Paris, den 15. Januar 2003). Centre Virtuel de la Connaissance sur l'Europe (CVCE). https://www.cvce.eu/en/obj/deutsch_franzosischer_ beitrag_zum_europaischen_konvent_uber_die_institutionelle_architektur_der_union_berlin_und paris_15_januar_2003-de-1a525f3e-3143-476c-9bea-1722f8250da9.html. Zugegriffen: 01. Dez. 2019.

Gauck, J. (2014). Deutschlands Rolle in der Welt - Anmerkungen zu Verantwortung, Normen und Bündnissen. Zeitschrift für Außen- und Sicherheitspolitik, 7(2), 115-122.

Haftendorn, H. (2001). Deutsche Außenpolitik zwischen Selbstbeschränkung und Selbstbehauptung. 1945-2000. Stuttgart, München: Deutsche Verlags-Anstalt.

Hellmann, G. (2002). Der „,deutsche Weg“. Eine außenpolitische Gratwanderung. Internationale Politik, 57(9), 1-8.

Hellmann, G. (2016). Zwischen Gestaltungsmacht und Hegemoniefalle. Zur neuesten Debatte über eine „,neue deutsche Außenpolitik“. Aus Politik und Zeitgeschichte, 66(28-29), 4-12.

Hellmann, G., Wagner, W., \& Baumann, R. (2014). Deutsche Außenpolitik. Eine Einführung. Wiesbaden: Springer VS.

Kundnani, H. (2016). The paradox of German power. London: Hurst \& Company.

Maull, H. W. (1990). Germany and Japan: The new civilian powers. Foreign Affairs, 69(5), 91-106.

Maull, H.W. (2014). „Zivilmacht“: Ursprünge und Entwicklungspfade eines umstrittenen Konzeptes. In S. Harnisch, \& J. Schild (Hrsg.), Deutsche Außenpolitik und internationale Führung. Ressourcen, Praktiken und Politiken in einer veränderten Europäischen Union (Deutsche Außenpolitik und internationale Ordnung) (S. 121-147). Baden-Baden: Nomos.

Meyer, J. (2003). Amendment Form. Art. I-46 Titel X (CONV 648/03) vom 2. April 2003. In Deutscher Bundestag/Referat Öffentlichkeitsarbeit (Hrsg.), Eine Verfassung für Europa. Der Europäische Konvent und der Deutsche Bundestag (Zur Sache 1/2003) (S. 1217-1218). Berlin: Deutscher Bundestag.

Möller, A., \& Rappold, J. (2012). Deutschland und der Europäische Auswärtige Dienst. Perspektiven einer Europäisierung der Außenpolitik. DGAPanalyse, (12). Deutsche Gesellschaft für Auswärtige Politik (DGAP). https://dgap.org/system/files/article_pdfs/2012-12_DGAPana_Moeller_Rappold_EAD. pdf. Zugegriffen: 01. Apr. 2020.

Müller-Brandeck-Bocquet, G. (2019). Gemeinsame Außen- und Sicherheitspolitik der EU: Neue Perspektive nach dem Referendum. In Die Rektoratskommission Studium Generale (Hrsg.), Europa Realität und Vision (S. 11-28). Heidelberg: Heidelberg University Publishing.

Müller-Brandeck-Bocquet, G., \& Rüger, C. (2015). Die Außenpolitik der EU. Berlin, Boston: De Gruyter. Oswald, S. (2018). Zehn Jahre Afrikapolitik. Zeitschrift für Außen- und Sicherheitspolitik, 11(4), 603-610. Petrov, P., Pomorska, K., \& Vanhoonacker, S. (2012). Introduction. The emerging EU diplomatic system: Opportunities and challenges after 'Lisbon'. The Hague Journal of Diplomacy, 7(1), 1-9.

Pilegaard, J., \& Kluth, M. (2012). The making of the EU's external action service: A neorealist interpretation. European Foreign Affairs Review, 17(2), 303-322.

Pleuger, G. (2002, 05. Nov.). "Double hat”. Working Document 17. The European Convention. Working Group VII - "External Action". http://european-convention.europa.eu/docs/wd7/4484.pdf. Zugegriffen: 01. Dez. 2019.

Rat der Europäischen Union. (2010, 26. Juli). Beschluss des Rates vom 26. Juli 2010 über die Organisation und die Arbeitsweise des Europäischen Auswärtigen Dienstes (2010/427/EU). EUR-Lex. https:// eur-lex.europa.eu/LexUriServ/LexUriServ.do?uri=OJ:L:2010:201:0030:0040:DE:PDF. Zugegriffen: 14. Jan. 2020. 
Rinke, A. (2016). Migration, Sicherheit, Wirtschaft. Afrika wird zu einer Priorität der deutschen und europäischen Politik. Internationale Politik, 71(6), 8-16.

Schmidt, V. A. (2005). Democracy in Europe: The impact of European integration. Perspectives on Politics, $3(4), 761-779$.

Simms, B. (2003). From the Kohl to the Fischer doctrine: Germany and the wars of the Yugoslav succession, 1991-1999. German History, 21(3), 393-414.

Simms, B. (2014). Europe. The struggle for supremacy. 1453 to the present. London: Penguin Books.

Steinmeier, F.-W. (2014, 01. Feb.). Rede von Außenminister Frank-Walter Steinmeier anlässlich der 50. Münchner Sicherheitskonferenz. Auswärtiges Amt. https://www.auswaertiges-amt.de/de/ newsroom/140201-bm-muesiko/259554. Zugegriffen: 01. Dez. 2019.

Vertrag über eine Verfassung für Europa, Art. I-28, III-296 (2004).

Wallace, W. (2005). Foreign and security policy. In H. Wallace, W. Wallace, \& M. Pollack (Hrsg.), Policymaking in the European Union (S. 429-456). Oxford: Oxford University Press.

Wiesner, C. (2018). Parteien, Europäisierung und die Politisierung der europäischen Integration. Eine vergleichende Betrachtung Deutschlands und Frankreichs. In L.H. Anders, H. Scheller, \& T. Tuntschew (Hrsg.), Parteien und die Politisierung der Europäischen Union (Vergleichende Politikwissenschaft) (S. 283-309). Wiesbaden: Springer VS.

Wouters, J., \& Duquet, S. (2012). The EU and international diplomatic law: New horizons? The Hague Journal of Diplomacy, 7(1), 31-49. 